\title{
A SYMMETRIC SURFACE MICROMACHINED GYROSCOPE WITH DECOUPLED OSCILLATION MODES
}

\author{
Said Emre Alper* and Tayfun Akin**** \\ *Middle East Technical University, Dept. of Electrical and Electronics Eng., \\ Ankara, Turkey. said@metu.edu.tr \\ **TUBITAK-ODTU-BILTEN, Middle East Technical University, \\ Ankara, Turkey. tayfun-akin@metu.edu.tr
}

\begin{abstract}
SUMMARY
This paper reports a new symmetric gyroscope structure that allows not only matched resonant frequencies for the drive and sense vibration modes for better resolution, but also decoupled drive and sense oscillation modes for preventing unstable operation due to mechanical coupling. The symmetry and decoupling features are achieved at the same time with a new suspension beam design. The gyroscope structure is designed using a standard three-layer polysilicon surface micromachining process (MUMPs) and simulated using the MEMCAD software. Measured results show that the drive and sense mode resonant frequencies are $28,535 \mathrm{~Hz}$ and $30,306 \mathrm{~Hz}$, respectively, which are in agreement with the finite element simulations. The small mismatch can be reduced further by applying different DC bias voltages to the drive and sense electrodes, improving the performance. The performance of the fabricated sensor is limited due to large parasitic capacitance between the proof mass and the substrate, nevertheless, measurements and calculations show that the sensor can sense angular rates as small as $0.37 \mathrm{deg} / \mathrm{sec}$ even in atmospheric pressure. A capacitive readout circuit for the sensor was also developed in a $0.8 \mu \mathrm{m}$ CMOS process, and the fabricated circuit detects capacitance changes smaller than $0.1 \mathrm{fF}$ with a sensitivity of $45 \mathrm{mV} / \mathrm{fF}$.
\end{abstract}

Keywords: Symmetric gyroscope, decoupled gyroscope, micromachined gyroscope,.

\section{INTRODUCTION}

Micromachined gyroscopes are quite popular in many applications such as camcorder stabilization, roll-over detection, and inertial mouse due to their very low cost. However, the rate sensitivity of these devices are wellbelow the conventional ring-laser and fiberoptic gyroscopes [1]. There are extensive studies on increasing the performances of the micromachined gyroscopes [2-6]. One method is to implement symmetric gyroscopes with matched resonant frequencies for the drive and sense vibration modes, so that gyroscope resolution is amplified with the mechanical quality factor of its sense resonance mode $[1,6]$. Some approaches try to match resonant frequencies by designing symmetric drive and sense suspension beams [2]; however, when the frequency mismatch between drive and sense modes is reduced, the mechanical coupling between them increases due to the nature of the suspension beams [3, 4], causing unstable device operation. To decrease mechanical coupling, various structures $[4,5]$ have been proposed where the drive and sense suspension beams are independent. However, it is not easy to match the resonant frequencies in these structures, since their suspension beams are not symmetric, and hence, process dependent.

This paper proposes a new gyroscope structure that solves problems of mechanical coupling and mismatched resonant frequencies at the same time by using a novel symmetric suspension beam design with decoupled vibration modes for stable operation.

\section{GYROSCOPE STRUCTURE}

Figure 1 shows the geometrical structure of the symmetric and decoupled gyroscope. The anchors of the structure are placed at the outermost corners and connected to the movable drive and sense electrodes with the help of suspension beams. This connection prevents mechanical coupling, since the oscillations of the two vibration modes do not affect each other. In addition, unlike the conventional approach, the suspension beams supporting the proof mass are not attached to the anchors directly, rather the proof mass is supported by the beams attached to the movable drive and sense electrodes, preserving the symmetry of the structure. 


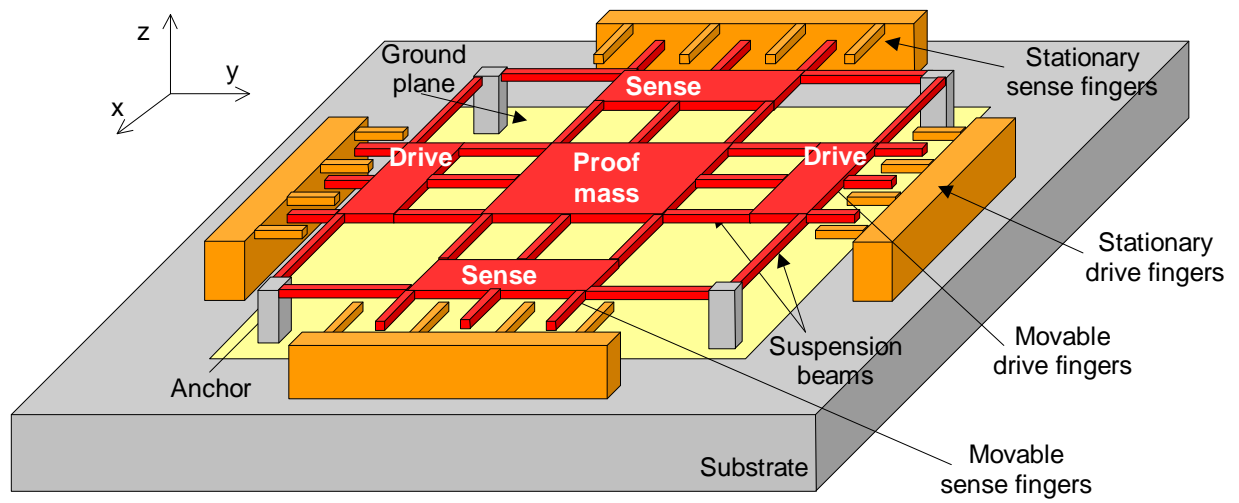

Fig. 1: Geometrical structure of the proposed gyroscope. The anchorage and suspensions are designed in such a way that the mechanical coupling between the drive and sense modes is suppressed and at the same time the structure has symmetric spring constants along $x$ and $y$ axes, achieving equal resonant frequencies of the two vibration modes.

The gyroscope operates according to the Coriolis coupling principle. The proof mass is vibrated along yaxis by applying suitable ac excitation between the stationary and movable drive fingers and a dc polarization voltage to the proof mass. When the substrate of the gyroscope is rotated around z-axis, the proof mass tends to vibrate along $\mathrm{x}$-axis due to Coriolis coupling. By measuring the amplitude of this induced vibration (sense mode), the angular rate of the applied rotation can be derived.

A common problem for vibrating gyroscopes is the cross-coupling between the drive and sense mode oscillations. The proposed structure solves this problem to a great extent due to its decoupled spring design for the two vibration modes, without destroying the symmetry of the device that is required for high rate sensitivity.

\section{FINITE ELEMENT SIMULATIONS}

Finite element simulations were used to verify the desired operation of the gyroscope. The simulations were performed with the MEMCAD 4 software package. Figure 2 shows MEMCAD modal simulation results for the drive and sense vibration modes. Resonant frequencies of the two modes are found to be equal and 33,154 Hz, due to the preserved symmetry. This would result in an improvement in the rate sensitivity by a factor equal to the quality factor of the sense vibration mode, which can be in the order of a few thousand for polysilicon structures in vacuum. These simulations also show that the vibration of a mode does not affect the other, i.e., mechanical decoupling between two modes is achieved with the novel structure of the suspension beams. This enhances the stability of the sensor and decreases the complexity of a feedback network.

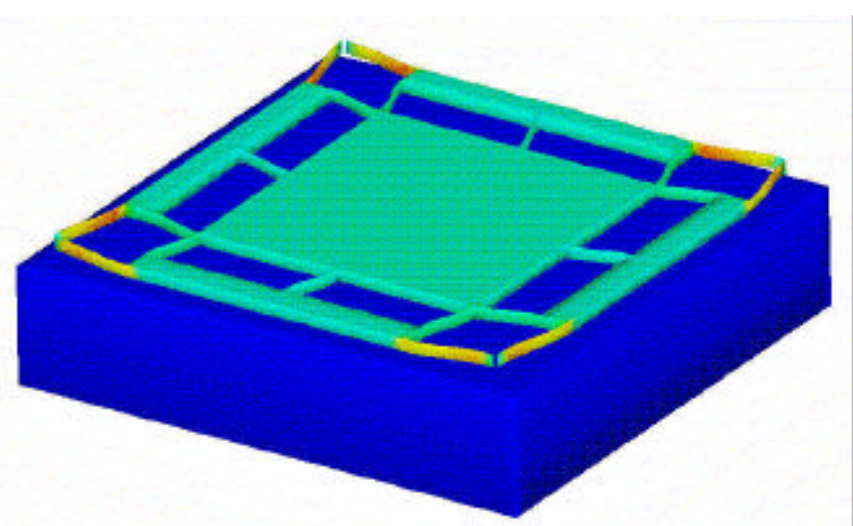

(a)

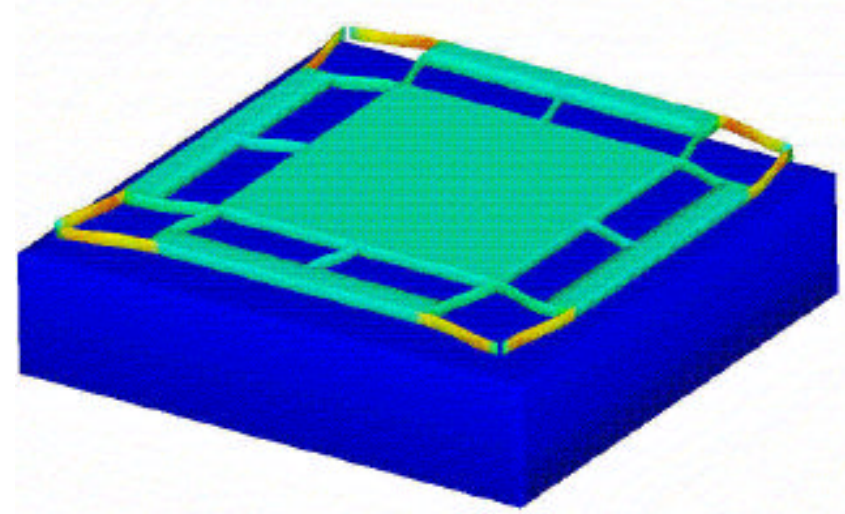

(b)

Fig. 2: MEMCAD modal simulation result for (a) the drive and $(b)$ the sense vibration modes. Resonant frequencies of the two modes are found to be equal and $33,154 \mathrm{~Hz}$, due to the preserved symmetry. The vibration of a mode does not affect the other, i.e., mechanical decoupling between two modes is achieved with the novel structure of the suspension beams. 


\section{IMPLEMENTATION AND TEST RESULTS}

The gyroscope is fabricated through a standard threelayer polysilicon surface-micromachining process provided by Cronos Inc. Figure 3 shows the SEM picture of the fabricated gyroscope which measures $1 \mathrm{~mm} \times 1 \mathrm{~mm}$. The thickness of the structural layer is $2 \mu \mathrm{m}$, defined by the foundry process. Due to the thin structural layer, the capacitances of the drive and sense modes are about $6.5 \mathrm{fF}$, limiting its performance. But, the performance is increased with the symmetric structure of the gyroscope, as discussed before.

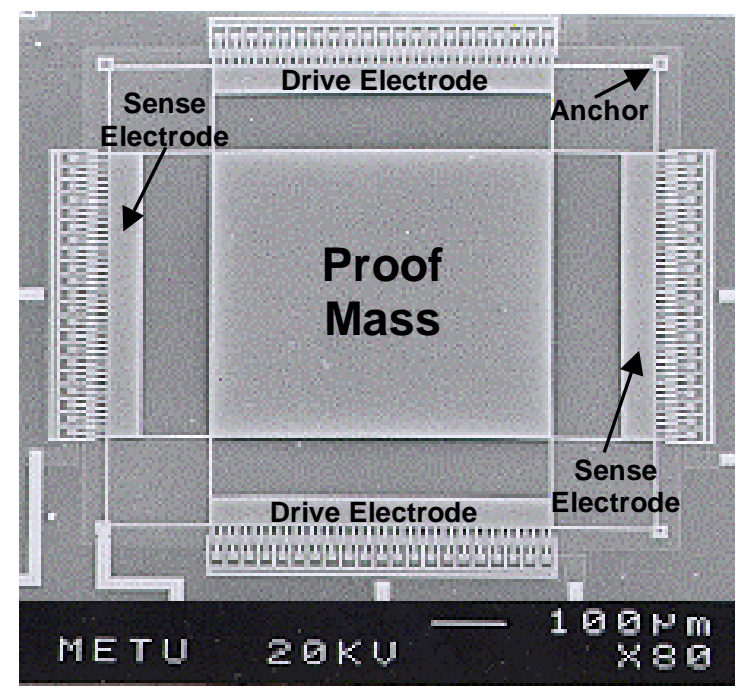

Fig. 3: SEM picture of the fabricated gyroscope which measures $1 \mathrm{~mm} \times 1 \mathrm{~mm}$.

A number of tests were performed to verify the device operation by determining the resonant frequencies and performance. Resonant frequencies of the drive and sense vibration modes of the planar gyroscope were measured by HP4395A Network/Spectrum Analyzer. These measurements were done by using a unity gain buffer circuit chip that was hybrid connected to the sensor. The buffer chip allows to monitor the high impedance output signal from the gyroscope. Figure 4 shows resonant frequency measurement results for (a) drive and (b) sense modes. Drive and sense mode resonant frequencies were measured as $28,535 \mathrm{~Hz}$ and $30,306 \mathrm{~Hz}$, respectively, with a $40 \mathrm{~V}$ dc bias (polarization) voltage applied to the proof mass. The mismatch comes from the non-uniform placement of the etch holes on the proof mass. The measured frequencies are slightly smaller than the simulated results since the simulations ignore the effect of comb fingers to shorten simulation time. The measurements were performed at atmospheric pressure and the results are expected to be improved in vacuum levels.

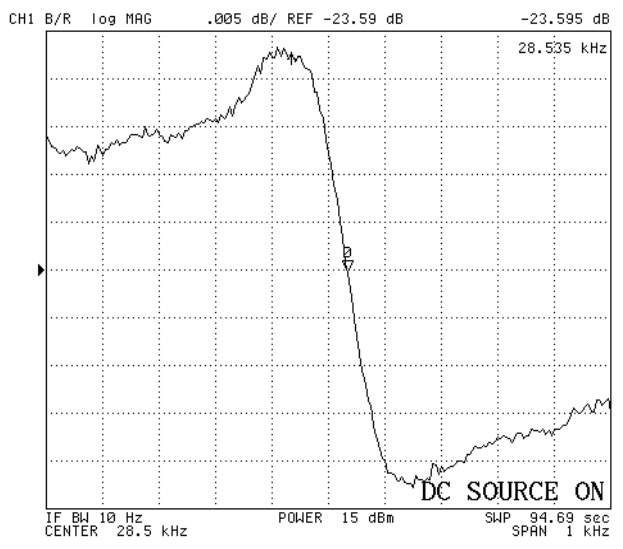

(a)

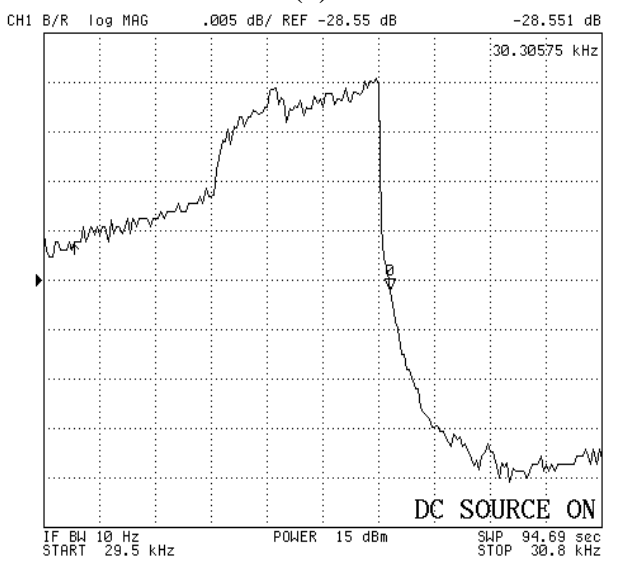

(b)

Fig. 4: Network analyzer measurement results for the resonant frequencies of the two vibration modes of the fabricated gyroscope, which are (a) 28,535 Hz for drive mode and (b) 30,306 Hz for the sense mode.

Figure 5 shows the resonant frequency shift versus dc bias voltage for the drive and sense modes. By applying different dc bias voltages separately to the drive and sense electrodes, the small mismatch between the resonant frequencies can be decreased further.

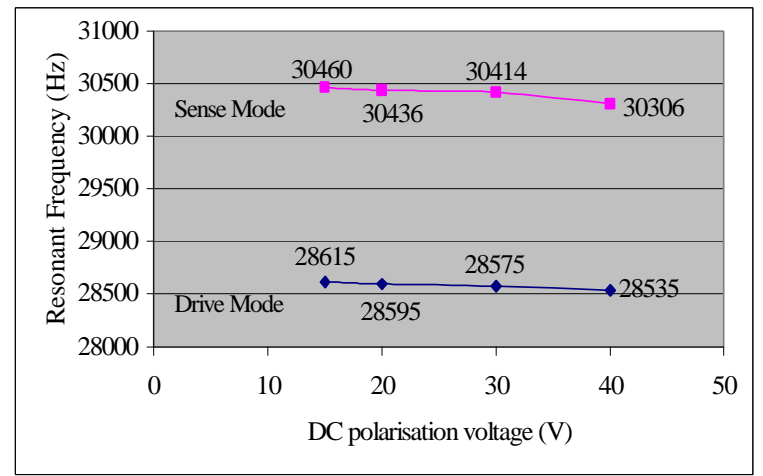

Fig. 5: Resonant frequency versus DC bias voltage for the drive and sense modes of the fabricated gyroscope. 
The resonant frequency measurements show that the amplitudes of the resonant peaks are quite low due to parasitic degradation. Parasitic capacitance at the high impedance node of the sensor is an addition of capacitances between the sensor mass, interconnects, bonding pads, buffer input, and the substrate. Figure 6 shows the parasitic model of the gyroscope output including the hybrid connection to the buffer chip. The parasitic capacitance at the high-impedance node of the sensor is measured using HP4294A Precision Impedance Analyzer and found to be around $3 \mathrm{pF}$, whereas the sensor capacitances are only 6-7fF. Obviously, the output signal of the sensor is degraded by more than two orders of magnitude due to the parasitic capacitances. Parasitics is a common problem for the capacitive sensors and can be effectively avoided using on-chip readout schemes with improved bootstrapping techniques [7], which is under consideration for future designs.

Although the performance of the sensor is highly limited with the parasitics, the symmetric gyroscope can still outputs $4 \mathrm{mV}$ per femtoFarad change in the sense capacitance. Theoretical calculations show that the gyroscope can sense rotation rates as low as $0.37 \mathrm{deg} / \mathrm{sec}$ in ambient, if the readout circuit noise can be kept below $0.1 \mu \mathrm{V}$ in a $30 \mathrm{~Hz}$ band, which is easily achieved with careful design. The sensitivity of the device is expected to be higher for operation in vacuum.

A capacitive readout circuit was also designed and fabricated in a standard $0.8 \mu \mathrm{m}$ CMOS process. The readout circuit is tested alone and verified to detect capacitance changes smaller than $0.1 \mathrm{fF}$, with a sensitivity of $45 \mathrm{mV} / \mathrm{fF}$. Detailed characterization of the hybrid-connected system in vacuum with a rate table is underway.

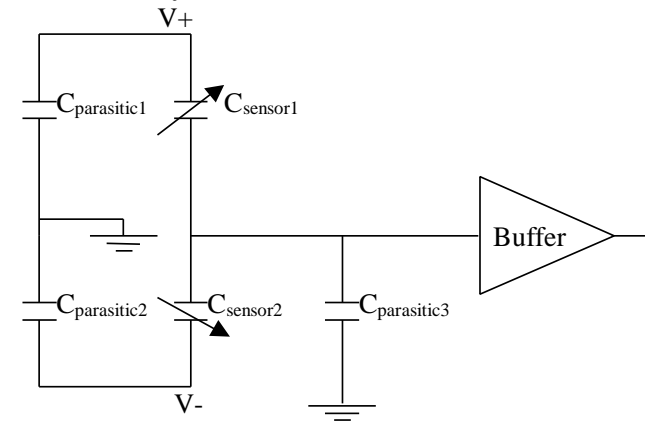

Fig. 6: Parasitic model of the high-impedance (output) node of the gyroscope. $C_{\text {parasitic } 3}$ is the dominant parasitic capacitance degrading the sensor output voltage. It can be effectively eliminated by using on-chip electronics.

\section{CONCLUSIONS AND FUTURE WORK}

A new symmetric gyroscope structure is presented that allows not only matched resonant frequencies for the drive and sense vibration modes for better resolution, but also decoupled drive and sense oscillation modes for preventing unstable operation due to mechanical coupling. The device operation is verified by FEM simulations using MEMCAD software, and the resonant frequencies of the drive and sense vibration modes are measured to be close to the simulated results. Measurements and calculations show that the structure can provide a resolution better than $0.37 \mathrm{deg} / \mathrm{sec}$ in ambient, which can be improved further by eliminating the parasitic capacitances and air damping effects. The developed structure can also be applied to high performance gyroscopes with advanced micromachining processes that have thicker structural layers. The device performance can be enhanced by fabricating the gyroscope with on-chip readout electronics and operation in vacuum.

\section{REFERENCES}

[1] N. Yazdi, F. Ayazi, and K. Najafi, "Micromachined Inertial Sensors," Proc. of the IEEE, Vol.86, No. 8, August 1998, pp. 1640-1659.

[2] S. S. Baek, Y. S. Oh, B. J. Ha, S. D. An, B. H. An, H. Song, and C. M. Song, "A Symmetrical Zaxis Gyroscope with a High Aspect Ratio Using Simple and New Process," MEMS'99, pp. 612617.

[3] H. Kawai, M. Tamura, and K. Ohwada, " Direct Measurement of Mechanical Coupling in Microgyroscope Using a Two-Dimensional Laser Displacementmeter," Transducers'99, pp.15661569

[4] Y. Mochida, M. Tamura, and K. Ohwada, "A Micromachined Vibrating Rate Gyroscope with Independent Beams for the Drive and Detection Modes," MEMS'99, pp. 618-623.

[5] W. Geiger, J. Merz, T. Fischer, B. Folkmer, H. Sandmaier, and W. Lang, "The Silicon Angular Rate Sensor System MARS-RR,” Transducers'99, pp. 1578-1581.

[6] F. Ayazi and K. Najafi, Design and Fabrication of a High-Performance Polysilicon Vibrating Ring Gyroscope, ” MEMS'98, pp.621-626.

[7] C. Lu, M. Lemkin, and B. E. Boser, "A Monolithic Surface $\quad$ Micromachined Accelerometer with Digital Output," IEEE J. Solid-State Circuits, Vol. 30, no. 12, pp. 13671373, December 1995. 